\title{
PUNKTZEIT: EL TIEMPO INVERTEBRADO. BREVE IDEA DEL TIEMPO EN LA CIENCIA Y TEORÍA LITERARIA DE FIN DE SIGLO
}

Víctor Borrero Zapata

In hoc punctum coniectus est, punctum est quod vivimus, in puncto fugientis temporis pendeo

Séneca

Time present and time past

Are both perhaps present in time future, And time future contained in time past. If all time is eternally present All time is unredeemable

T. S. Eliot

Niego, en un número elevado de casos, lo sucesivo; niego, en un número elevado de casos, lo contemporáneo también. El amante que piensa: «Mientras yo estaba tan feliz, pensando en la fidelidad de mi amor, ella me engañaba», se engaña: si cada estado que vivimos es absoluto, esa felicidad no fue contemporánea de esa traición.

La voz de Borges, a quien pertenecen estas palabras, anticipa ya en los años cincuenta un debate que a fin de siglo y de milenio ha intensificado inexorablemente: el debate sobre el tiempo, su medida, nuestra percepción en estos tiempos de aceleración y de cambio.

Borges, en su Nueva refutación del Tiempo, que según él mismo reconoce es tan antigua como la flecha de Zenón, añade:

Cada instante es autónomo. Ni la venganza ni el perdón ni las cárceles ni siquiera el olvido pueden modificar el invulnerable pasado. No menos vanos me parecen la esperanza y el miedo, que siempre se refieren a hechos futuros; es decir, a hechos que no nos ocurrirán a nosotros, que somos el minucioso presente (...) No hay esa historia <del universo >, como no hay la vida de un hombre, ni siquiera una de sus noches; cada momento que vivimos existe, no su imaginario conjunto (BORGES, 1989: 356).

Esta clarísima negación del tiempo entendido como sucesión de hechos ordenados y acumulados en una línea progresiva de lo que fue, lo que es y lo que será; y la afirmación 
en su lugar de un tiempo del instante presente, del instante vivido nos introduce muy certeramente en el concepto de Punktzeit, tiempo puntual, que es una forma más de posmoderna desvertebración.

Punktzeit, que a partir de ahora traduciré como tiempo puntual, ha sido introducido por el filósofo Paul Virilo como una nueva forma de percepción del tiempo propia de la edad posmoderna. Este concepto expresa teóricamente la disolución del tradicional tiempo lineal, la disgregación de la jerarquía del continuum temporal en unidades mínimas y aisladas, en instantes mínimos de experiencia vital, quizá también poética ${ }^{1}$.

Esta nueva forma de percepción temporal habría nacido al abrigo de dos circunstancias:

Por un lado, de las últimas transformaciones sociales en sociedades industrializadas, en la nueva urbe, derivadas de la mundialización de sus estructuras (recordemos que nos encontramos en la sociedad de la telemática, de las redes de flujo continuo y simultáneo de información en constante aceleración, del teletrabajo, del teledinero).

Por otro, es importante la influencia en la configuración de este concepto de la mutación de las mentalidades, los hábitos y los valores occidentales en su relación con el tiempo y el espacio $^{2}$ como consecuencia de la revolución científica y técnica (sobre todo en la física cuántica y la astrofísica; en la neurofísica, la psicología y la fisiología; y en la ingeniería de las telecomunicaciones.

No nos parece, pues, aventurado especular en este sentido que la percepción subjetiva del tiempo camine pareja, de alguna manera, a su medición científica, que el horizonte del conocimiento humano, del saber objetivo por medio de la razón (en su vertientes científica y especulativa), influye y se ve irremediablemente influido por el modo particular de sentir del hombre, de percibir e interpretar subjetiva y sensitivamente, ya sea en su dimensión individual o social, la realidad que le rodea.

Así, partiendo de este sencillo razonamiento, y aceptando la distinción de Bergson entre el tiempo objetivo o tiempo mensurable ("temps") y el tiempo interior o tiempo percibido ("durée"), podríamos inferir que la imagen del tiempo en su larga historia es una suma dialéctica e irregular de medición objetiva más percepción subjetiva.

1 «Der französische Geschwindigkeitstheoretiker Paul Virilo versucht, den Nachweis zu führen, daß wir im Machtsystem der Beschleunigungen, also im Zeitalter der elektronischen Medien und künstlichen Intelligenzen, an einer neuen Epochenschwelle stehen. Folgt man Virilio und seinen Adepten, so setzt sich in der modernen Informations- und Computer-Gesellschaft, die neue, unvorstellbare Räume erschließt, auch eine qualitativ neue Zeit-Erfahrung durch: eben die "Punktzeit" Relativitätstheorie, Quantenmechanik, Informationstheorie und Kybernetik antizipieren theoretisch die Auflösung des linearen Zeit-Kontinuums (...). An einen Terminus wie »Punktzeit « lagern sich aber noch andere schillernde Konnotationen an. Denn er erinnert zugleich an den lyrischen Zeit-Punkt, den poetischen Augenblick, die »kleinste Erlebniseinheit, die Einzelnes scharfrandig herauschneidet aus dem Allzuvielen " (Walter Höllerer). In der Evokation des poetischen Augenblicks widersetzt sich auch noch heute das schreibende Ich der Wahrnehmungs-Zerstreeung, der Flut der heranstürmenden Bewußtseinsreize» (RIHA, 1996: 305-306).

${ }^{2}$ «La televisión produce algo mucho más profundo que la invasión de la intimidad o el consumo productivo (...): también genera una urbanización del tiempo de los ciudadanos. (...)» (ECHEVERRÍA, 1995: 102). 
No es casual, pues, que en la Edad Antigua la concepción del tiempo de hebreos y griegos, amparada por una visión teocéntrica y mitológica del mundo y limitada por su horizonte de conocimiento, por su creencia en el destino, tuviera un carácter absoluto y cíclico, calculado por el movimiento de los cuerpos celestes, por el día y la noche, por la luz y la oscuridad, a la vez que rítmico, medido por los latidos ("reghá") del corazón (FERRATER, 1981: 794-810).

Tampoco es una relación azarosa la que vincula el descubrimiento del reloj mecánico (1271) en la Baja Edad Media con la instauración de una primera medición homogénea, unificada del tiempo social mediante la instalación de relojes en las torres y campanarios de las plazas públicas de las primeras urbes ${ }^{3}$. O que, a partir del Renacimiento y los descubrimientos de los Copérnico, Kepler, Galileo y Newton quede fundada una nueva concepción científica del tiempo, del mundo y de sus leyes físicas. El tiempo, desde entonces, será entendido por el hombre, además de cómo una categoría absoluta, como una categoría matemática, aprehensible por la razón humana. Una primera tentativa de racionalización científica del tiempo que atiende también a otros intereses que son, digámoslo así, "sociales": los pragmáticos del maridaje entre el tiempo y el dinero, que a partir de entonces será indisoluble.

La edad ilustrada confirma una concepción lineal del tiempo. El paso de la comprensión del tiempo como un fenómeno cíclico a su explicación como un fenómeno progresivo marca también un punto de inflexión entre dos formas radicalmente opuestas de comprender el universo y la Historia. Quedan atrás las teorías mecanicistas de Newton para dar paso al optimismo finalista de Kant: la Historia es, ante todo, progreso del hombre emancipado ${ }^{4}$.

Por último, tampoco sorprende que la era de la industrialización, en el siglo XIX, continuada en el siglo XX, haya sido la encargada de abrir el camino, de modo irreversible, hacia una visión cada vez más relativizada del tiempo. El tiempo social, por las innovaciones técnicas en transportes y comunicaciones, sufre una aceleración constante, tendente a desnaturalizar sus percepciones tradicionales y a convertirse en un presente ubicuo $^{5}$, en un nunc stans. Por otro lado, desde su observatorio científico, Einstein, en el primer cuarto de siglo, desarrolla las teorías de la relatividad especial y general, modificando drásticamente la ecuación de Newton en la que el tiempo aparecía como una constante absoluta y estática por cuanto estaba apoyada en una explicación teísta del origen del universo. A partir de ahora, el tiempo, desde el punto de vista físico, será una

${ }^{3}$ «Am Anfang der Entwicklung des die abendländische Kultur dominierenden neuzeitlichen Zeitbegiffs stand eine einfache Vorrichtung, die es erlaubte, Uhrwerke mit dem Glockenspiel von Kirch- und Rathaustürmen zu koppeln. Die Wirkung dieser Einrichtungwar nicht zu überschätzen; leitete sie doch die Entwicklung von einer anthropzentrischen zu eiener chronozentrischen Lebensform ein. (...) Durch die mechanische Koppelung der Rädeuhren mit den Glocken von Kirch- und Rathaustürmen weiß ab sofort jeder Bürger, was die Stunde geschlagen hat.»( MECKE, 1990: 1).

${ }^{4}$ Cfr. Sahmel.K.-H.: "Momo oder: Pädagogisch relevante Aspekte des Problems der Zeit”

${ }^{5}$ Cfr. Ramos, R.: "El presente ubicuo: tiempo y sociedad en una época de crisis". 
coordenada dinámica e inseparable del espacio, una cuarta dimensión dell binomio espaciotiempo ${ }^{6}$, en un universo inestable y en continua expansión.

Paralelamente, en este mismo siglo XX, la filosofía más especulativa, la metafísica, ofrece su propio modo de resistencia al progreso del conocimiento objetivo (de la ciencia y la técnica) del Tiempo. A una ciencia que alcanza a reconocer el paso del tiempo en la materia en dimensiones inauditas para los sentidos del hombre. Las conclusiones de la metafísica postularán con Bergson (a fines del XIX), Husserl, Russel (y su stream of concioussness) y Heidegger en Sein und Zeit, un paso del tiempo interior, durativo, específicamente humano y verdadero, un tiempo como flujo de la conciencia opuesto a un tiempo físico, ajeno al hombre.

En resumen, la historia de la medición del tiempo y de su percepción hasta el tiempo puntual posmoderno pudiera glosarse en una alternancia de tres modelos de concepción radicalmente distintos:

Por un lado, el tiempo como ciclo (de la vida, del movimiento de los astros, de las estaciones) eterna e idénticamente repetible. Por otro, el tiempo como categoría teleológica, como sucesión lineal de sucesos dirigidos hacia un fin (el ideal de perfección, la muerte, la utopía, el encuentro con Dios). Y, por último, el tiempo como presente ubicuo e invertebrado, puntual, del instante vivido, que contiene en sí la memoria y la expectativa como emociones ficticias del momento presente.

De entre ellas habremos de quedarnos aquí con esta última: el tiempo, y, por ende, la Historia, como un fenómeno puntual. Esta última perspectiva, que corresponde a la noción posmoderna del tiempo, surge como distanciamiento consciente ( $y$, de alguna manera, irónico) de su concepción moderna (es decir, lineal y progresiva), inspirada en un discurso ilustrado unitario y universal que había sido resucitado en un primer momento por marxistas y, finalmente por los neomarxistas de la Escuela de Frankfurt.

No obstante, la enunciación del fin de la modernidad (Nietzsche), de un nuevo pensamiento débil (Vattimo) desjerarquizado, descentralizado, la muerte de los grandes relatos de la Edad Moderna (Lyotard) son probablemente, antes que especulativas teorías metafísicas, las conclusiones de filósofos culturales y de la ciencia a los avances científicotécnicos de una nueva era, la era de la comunicación global, que introduce notables modificaciones conceptuales en nuestra teoría del conocimiento.

De un lado, el relativismo perspectivista. La coordenada tiempo no puede ser imaginada como una abstracción independiente del espacio. Es decir, la división del tiempo en unidades cada vez más pequeñas, nanométricas, su aceleración, debe provocar ineludiblemente modificaciones en el concepto espacial. Una de ellas es la apertura definitiva de perspectivas para la descripción de un movimiento, en el sentido en que en

\footnotetext{
6 "Un suceso es algo que ocurre en un punto particular del espacio y en un instante específico del tiempo. Por ello, se puede describir por medio de cuatro números o coordenadas. (...) En relatividad, no existe una distinción real entre las coordenadas espaciales y la temporal, exactamente igual a como no hay ninguna diferencia real entre dos coordenadas espaciales cualesquiera" (HAWKING, 1988: 51).
} 
ningún caso éste puede ser descrito desde una única posición universal ${ }^{7}$. Esta conclusión, producto del desarrollo de Heisenberg de la teoría de la Relatividad de Einstein, ha dado lugar a otorgar a la relatividad de la perspectiva para la escrutación de conclusiones científicas un lugar central tanto en la enunciación de teorías del conocimiento ${ }^{8}$ como en la misma labor artística.

Al mismo tiempo, incluso, emerge la concepción de una imagen del mundo como fruto de nuestros constructos cognitivos. La ciencia comprueba que la imagen de la realidad física tal cual se aparece ante nuestros sentidos es resultado de una prefiguración por parte de los mismos, y desaparece la concepción de un mundo físico estructuralmente articulado conforme a una idea superior. En su lugar nace la concepción de un cosmos formado por micro- y macrosistemas irregulares y dinámicos de retículas. ${ }^{9}$

Por otra parte, retomando nuestro tema del tiempo, y en directa relación con lo dicho anteriormente, la técnica de los medios consigue crear una disimetría entre el tiempo real de la emisión (más veloz) y el tiempo de la recepción (aparentemente más lento). Es decir, la técnica se ha ocupado, como el famoso cuadro de Durero describe, o la técnica pictórica puntillista representa, de descomponer la información natural (visual, auditiva, intelectiva) en unidades mínimas codificadas (en pixels como diminutos haces de luces proyectados o códigos binarios), en puntos, que "dan la impresión" por la forma de su conjunto y su velocidad de sucesión, por su frecuencia, de ser idénticas a la realidad retratada, si bien su composición real es de naturaleza completamente diversa, "desnaturalizada". Una imagen moderna, la de una pantalla de televisión, de ordenador, estará compuesta en realidad, no por una, sino por 24 imágenes por segundo que cobran por esta operación apariencia de movimiento. Lo que aparece ante nuestros sentidos como imágenes diáfanas de movimiento, por esta extraña paradoja del tiempo y la técnica, no es más que la operación de una perfecta descomposición, una operación que ofrece el ideal de la comunicación a tiempo real a través de la producción de un tiempo falseado. Esta creación por el ser humano de mundos aparentes, virtuales, inventará una nueva noción del tiempo y el espacio.

Esta anisocronía entre tiempos de emisión y percepción en procesos comunicativos es aceptada como una prueba de hecho en los procesos de percepción de la mente humana. Recientes estudios han demostrado que tampoco para nuestro sistema neurológico existe

7 "Entweder Ort oder Geschwindigkeit können bestimmt werden, nicht beides zugleich. In der Raum-Zenit der Quantenmechanik kann der Beobachter, der hier Bewegungen mißt, keinen neutralen Posten mehr beziehen. Solcher Perspektivismus gilt nicht bloß für die Mikroräume, er bestimmt Wahrnehmumgsprozesse generell."(GENDOLLA, 1992: 86).

${ }^{8}$ Teorías del conocimiento (o de la cognición) como la Teoría de los Sistemas (Schmidt) o literarias modernas como la Hermenéutica (Gadamer) o la Estética de la Recepción (Jauss) otorgan a la perspectiva del obvservador un papel fundamental en la descripción de sus respectivos objetos de estudio.

9 “An den Polen der Bewegung, im Kleinsten wie im Größten, im Mikro- wie im Makrokosmos erscheinen jeweils Punkte, letzte Punkte, die sich dann wieder als vorletzte herausstellen: [...] Körper, Flächen und Linien sind nur Zusammensetzungen solcher Punkte, gewebt in den Koordinaten eines Rasters, das selbst wieder aus Punkten zusammenfügt wurde, Kreuzungspunkte eines anderen Rasters. Der Wahrnehmungsprozeß ist das Herstellen von Rasterungen, in denen dann etwas als Objekt erscheinen kann." (GENDOLLA, 1992: 87). 
una única dimensión temporal. Conocemos, a partir de las investigaciones de Florey y Libet, que los sucesos que acontecen en el exterior, procesados por nuestros aparatos sensitivos, son registrados a diferentes velocidades según su recorrido a través de los nervios sensitivos hasta llegar al cortex sensorial en el cerebro. Es decir, nuestra percepción del tiempo de la duración de los acontecimientos más inmediatos no será idéntica a la duración real de los mismos, ni el momento en que nuestro cerebro los perciba será el mismo que en el que se ha producido el contacto real con ellos.

Por último, y desde el punto de vista de la astrofísica, existe constatación científica del carácter irreversible del tiempo, rectificando de este modo las primeras conclusiones de la Teoría de la Relatividad Especial. Así, las posibilidades en un principio abiertas a un universo tan expansivo como contractivo que hubieran conllevado una "flecha del tiempo" en dirección al futuro tan posible como hacia el pasado (es decir, la posibilidad teórica de recordar el pasado desde el futuro o el futuro desde el pasado) se cierra definitivamente a la primera de ellas por la segunda ley de la termodinámica, según la cual el universo avanza en el sentido del tiempo (esto es, del pasado al futuro) a partir del cual el orden deviene entropía.

Esta podría ser la imagen que la ciencia y la técnica de la edad posmoderna han colaborado en definir sobre el tiempo: un tiempo que sólo existe como unidad en abstracto, un tiempo asincrónico y unidireccional, un tiempo tetradimensional en relación indisoluble con el espacio.

Si bien resultaría útil desde un punto de vista metodológico acoger la esperanzadora propuesta de Fraser de inaugurar una nueva rama del saber denominada "cronosofía" con carácter interdisciplinar, con sus tres ramificaciones principales, dedicadas respectivamente a los problemas del pensamiento, de la naturaleza y del hombre en relación con el tiempo ${ }^{10}$, parece prematuro un análisis de la manifestación literaria bajo esta nueva perspectiva sin antes verse razonablemente cumplidas todas las condiciones de convergencia de las diferentes parcelas de conocimiento en torno a este problema. Por esta razón, a continuación voy a resumir la posición que algunas escuelas, corrientes o teorías literarias destacadas en esta mitad del siglo han adoptado hacia el problema temporal del relato. De cómo lo han problematizado, y hasta qué punto se han visto afectadas sus posturas en torno al tiempo por la incorporación de nuevas perspectivas filosóficas, de nuevos avances técnicos, de nuevos conocimientos científicos.

Uno de los puntos de discusión preponderantes en las teorías literarias de la actualidad es si el cambio de paradigma que en todos los campos del saber parece estar gestándose por la incorporación de nuevos conocimientos científico-técnicos habrá de provocar en el estudio de la obra literaria un cambio de perspectiva respecto a las categorías espaciotemporales en favor de una espacialización. En este debate el tiempo dejaría de ser considerado como el elemento vertebrador de la obra literaria y su función organizadora la ocuparían sus valores topográficos.

${ }^{10}$ Cfr. FRASER (1981: 582-593; 1987: 5-35). 
FISCHER-LICHTE (1988: 15-18) ejemplifica la problemática de la espacialización de las perspectivas de estudio del fenómeno literario en seis teorías literarias que de una manera $\mathrm{u}$ otra se ven afectadas por esta circunstancia: las teorías posestructuralistas o deconstructivistas, la teoría de la intertextualidad, la estética de la recepción, la teoría semiótica, la teoría narratológica y las teorías del drama y el teatro.

En las teorías posestructuralistas, en opinión de la autora, el cambio de paradigma hacia la espacialización del enfoque ya se ha consumado, dado que una oposición a la concepción moderna o neomarxista del tiempo literario implica la renuncia a la linealidad, a la sucesión continua. En su lugar, el posestructuralismo propone un enfoque basado en una "estética del espacio" en un primer nivel, sin renunciar, en un segundo plano a las informaciones textuales temporales.

Fischer-Lichte considera que a la estética de la recepción, por el contrario, le resulta más difícil sustituir una estética del tiempo por otra del espacio, dado que su tiempo y su espacio son el tiempo y el espacio de las comunidades interpretativas, que son el eje de su programática.

Para la aproximación semiótica a la manifestación literaria, donde la teoría de los polisistemas ocupa una posición preponderante, la distinción entre el espacio y el tiempo es más patente. El espacio, desde el Laokoon de Lessing, es la categoría propia del sistema de signos de exposición global y que, por esta razón, es el espacial por excelencia: la pintura; mientras que la temporal es la propia de sistemas de signos lineales, es decir, netamente temporales: la literatura. Todo ello si nos circunscribimos a su recepción visual. En el caso de la recepción auditiva, la clasificación de sistemas de signos temporales y espaciales sería otra muy diferente: más temporales serían aquellos signos lingüísticos transmitidos por la voz o por sonido, como la lengua oral; más espaciales serían los sistemas de signos escritos.

En lo tocante a la teoría narrativa, la autora considera que es la perspectiva teórica donde más queda de manifiesto el tránsito del enfoque temporal al espacial. Este cambio puede percibirse no sólo en el objeto de estudio en sí, el texto narrativo, sino, incluso más patentemente en la teoría narratológica ${ }^{11}$ que considera el texto como una realidad esencialmente sincrónica, cuyo sentido emana a través de una red de relaciones antes que mediante un proceso histórico o de cambio. Esto, añade Fischer-Lichte, queda avalado por su propia terminología, que inventa conceptos como "superficie y profundidad", "dentro y fuera", "linearidad", "intersección" y "distancia", que son eminentemente espaciales.

Las teorías del drama y el teatro centran también su atención en las categorías espaciotemporales, dado que son de gran utilidad para distinguir entre el drama como obra literaria o como representación teatral. De este modo, en el drama-texto habrá de predominar el plano espacial, mientras que la preponderancia del plano temporal será más propia, en el marco de esta comparación, de dramas representados.

11 "It is common to find time (the temporal ordering of events) stressed in work on narrative (...). However, I want to stress the centrality of a non-temporal "spatial-poetic function" in narrative, a fuction that I argue is a core aspect of human sense-making” (GEE, 1996: 23). 
Sin embargo, en la teoría de la intertextualidad se puede percibir verdaderamente, según Fischer-Lichte, el cambio paradigmático del concepto temporal al espacial. La referencia a Bachtin es a partir de entonces necesaria. El concepto de cronotopos, pese a su reciente redescubrimiento a partir del ingente legado de Bachtin sobre teoría de la obra narrativa, no es contemporáneo. Ya en los años 30 (sólo quince años después de la formulación de la Teoría de la Relatividad General) menciona en sus escritos la posibilidad de una unidad que aglutine las propiedades temporales y espaciales de un texto y sus relaciones dentro de él o con otros textos. Aunque hasta 1973 no publicará las conclusiones sobre esta teoría en su artículo"Formen der Zeit und des Chronotopos im Roman" en una labor continuada de actualización y redefinición hasta su aproximación a la Estética de la Recepción en el análisis del concepto de Cronotopos desde la perspectiva del oyente y el lector.

Bakhtin (también Bachtin, o Bajtin) propone con su concepto de cronotopos una conciliación indisociable entre el espacio y el tiempo de la obra literaria. Tiempo y espacio forman una unidad, desde la cual se analizarán las relaciones de espacio y tiempo intratextuales y también extratextuales, de manera que "el diálogo de un texto con otros puede ser entendido como una espacialización de conceptos dialógicos previamente determinados por el tiempo"(BAKHTIN, 1986:15). El concepto de cronotopos, basado en la teoría de la relatividad de Einstein y en su concepto de espacio-tiempo tetradimensional supone una prueba de la interacción de la filosofía, la ciencia, la técnica y las teorías literarias, de la influencia mutua entre ciencia y pensamiento.

NETHERSOLE ( 1988: 59-65) señala que el concepto de cronotopos de Baktin podría considerarse como el punto de inflexión entre un signo temporalista de la teoría literaria (historicistas, desde Herder, Scherer, Lessing y las escuelas marxistas) hacia una espacialización, y la creación de una poética del espacio iniciada por teorías atemporalistas, como el formalismo ruso, el estructuralismo francés y afirmada por el posestructuralismo y las tendencias posmodernistas, es decir, la poética de fin de siglo y de milenio habrá de consistir en una estética de relaciones sincrónicas entre textos, y sistemas antes que en una estética de las transformaciones sucesivas.

En este extenso itinerario hemos descrito un repaso de la historia del tiempo observada desde la ciencia, la técnica, especialmente en el siglo XX, y cómo distintas teorías literarias han estudiado el fenómeno literario. La literatura tampoco será ajena a estos cambios en el modo de entender y conceptualizar el tiempo. En ella hallarán espacio de expresión las distintas maneras de aproximación a la permanente complejización del tiempo, a su aceleración constante y su desmenuzamiento en unidades mínimas, a su valor relativo y multidimensional descubierto por científicos, taxonomizado por filósofos e incorporados a las distintas teorías literarias en sus respectivos órdenes programáticos por los teóricos de la literatura, como acabamos de apreciar.

Esa aproximación creativa al tiempo en sus múltiples dimensiones, fundamentalmente en el ámbito de la narrativa, podrá realizarse de distintos modos. El escritor podrá reproducir con diferentes técnicas las dimensiones temporales recién descubiertas. Pero también podrá aventurarse a transgredirlas, a subvertir la realidad temporal real a través de una realidad ficcional, la propia realidad de la obra literaria. 
En este punto se detiene nuestra exposición teórica sobre la definición del tiempo en nuestra era, de sus distintos modos de conceptuarlo -medirlo y percibirlo-. En resumen, se podría afirmar, ateniéndonos con una mirada amplia e interdisciplinar a la ingente cantidad de aportaciones sobre el tema, que en este tramo final del milenio, Punktzeit representa de una manera metafórica nuestra apreciación del tiempo. Un tiempo material porque existe en tanto la materia y su observador existan; un tiempo relativo e invertebrado porque su medición es la medición de quien la realiza y porque su organización es "temporal"; es también -y sobre todo- Punktzeit un tiempo que es territorio del espacio, y cuyo valor espacial seduce a muchos de quienes se acercan a él desde nuestra contemporaneidad. Ese valor espacial en literatura puede traducirse en una intensa indagación del momento presente, una ralentización del instante puntual del individuo como alternativa a los infinitesimales tiempos puntuales en que el progreso técnico ha multiplicado el tiempo común, el tiempo de todos, una ralentización de nuestro tiempo presente como refugio también ante su imparable proceso de aceleración social.

\section{BiBLIOGRAFÍA:}

BAKHTIN, M. (1986) Untersuchungen zur Poetik und Theorie des Romans, Berlín/Weimar.

BORGES, J.L. (1989) "Nueva refutación del tiempo", Obras Completas, Barcelona: Círculo de Lectores.

ECHEVERRÍA, J. (1995), Cosmopolitas domésticos, Barcelona: Anagrama.

FERRATER MORA, J. (1999), Diccionario de filosofía de bolsillo, Madrid: Alianza Editorial.

FISCHER-LICHTE, E. (1988), "The shift of the paradigm: from time to space? Introduction", Space and boundaries in literature, München: Iudicium-Verlag.

FRASER, J.T. (1981) "The Study of Time", The voices of time. A cooperative survey of man's views of time as expresed by the sciences and by the humanities, Amherst: The University of Massachussets Press, págs. 582-593.

FRASER, J.T. (1987), "Saliendo de la caverna de Platón: la historia natural del tiempo", Revista de Occidente, págs. 5-35.

GEE, J.P. (1996), "A Spatial-Poetic Approach to Narrative". En: Perspectives on Narratology. Papers from the Symposium on Narratology, Frankfurt am Main: P.Lang.

GENDOLLA, P. (1992) Zeit. Zur Geschichte der Zeiterfahrung. Vom Mythos zur «Punktzeit», Köln: Dumont.

HAWKING, S. W. (1988) Historia del tiempo. Del Big Bang a los agujeros negros, Barcelona: Círculo de Lectores.

LYOTARD, J.-F. (1994), Das postmoderne Wissen, Ein Bericht, Wien. 
MECKE, J. (1990) Roman-Zeit: Zeiformung und Dekonstruktion des französischen Romans der Gegenwart, Tübingen: Narr.

NETHERSOLE, R. (1988), "From Temporality to Spatiality: Changing concepts in Literary Criticism" Space and boundaries in literature. München: Iudicium Verlag, págs. 59-65.

RAMOS, R, "El presente ubicuo: tiempo y sociedad en una época de crisis", Revista de Occidente, págs. 96-107.

RIHA, K. (1996), “Zur Literatur der achtziger Jahre. Ein Situationsbericht (1988). Nebst Postkriptum 1995: Wendeliteratur, ein Schlaglicht”, Pluralismus und Postmodernismus: Zur Literatur und Kulturgeschichte in Deutschland 1980-1995. Helmut Kreuzer (Ed.), Frankfurt am Main: Lang, págs. 305-6.

SAHMEL, K.-H. (1988), "Momo oder: Pädagogisch relevante Aspekte des Problems der Zeit", Pädagogische Rundschau. Monatschrift für Erziehung und Unterricht, 42, págs. 403-419.

VATTIMO, G. (1985) La fine della modernitá, Torino.

WEGNER, M. (1988) "Zur Chronotopostheorie Michail Bachtins", Space and boundaries in literature. Proceedings of the congress of the international comparative literature association, München: Iudicium Verlag, págs. 36-42. 